\title{
Hubble operator in isotropic loop quantum cosmology
}

\author{
Golam Mortuza Hossain \\ The Institute of Mathematical Sciences, Chennai 600 113, \\ India \\ email:golam@imsc.res.in
}

October 25, 2018

\begin{abstract}
We present a construction of the Hubble operator for the spatially flat isotropic loop quantum cosmology. This operator is a Dirac observable on a subspace of the space of physical solutions. This subspace gets selected dynamically, requiring that its action be invariant on the physical solution space. As a simple illustrative application of the expectation value of the operator, we do find a generic phase of (super)inflation, a feature shown by Bojowald [1] from the analysis of effective Friedmann equation of loop quantum cosmology.
\end{abstract}

Pre-print No. IMSc/2003/06/11

\section{Introduction}

Symmetry reduction [2, 3] at the quantum level of loop quantum gravity [4, 5, 6] has been applied to the field of cosmology with notable success. The key result of this approach i.e. loop quantum cosmology is the resolution of classical cosmological singularity [7. Apart from this, the consideration of effective Friedmann Equation due to small volume modification of kinetic term of the matter fields leads to a phase of super-inflation with graceful exit [1]. But there are lots of major issues yet to be clarified. One such issue is the construction of physical observables [8, 9] in the loop quantum gravity. This problem stems directly from the fact that defining physical observables in general relativity itself is nontrivial as the physical observables are required to be gauge invariant objects. Consequently in the quantum theory the corresponding operators are required to commute with all the constraints of theory at least on the space of physical solutions. But the problems of the quantum theory in this regards remains as hard as of the classical theory if not more. Naturally this shortcoming is reflected in the loop quantum cosmology. But in the case of loop quantum cosmology one works with a much simpler setting compared to that of full theory. In particular only the Hamiltonian constraint needs to be addressed. So one expects that this problem will be easier to handle in the case of loop quantum cosmology.

In this paper, from a general perspective, we emphasize that a Dirac observable of a dynamical theory, to be physically relevant, must be localized [10] in an appropriate frame of reference. In the case of isotropic loop quantum cosmology, we show that the localization is no longer an option for construction of Dirac observables rather it is the only option. In other words, given a kinematical operator, one cannot construct the corresponding Dirac 
observable in the isotropic loop quantum cosmology without localization. Further we show that the construction of a Dirac observable allows one to choose only a subspace of the space of physical solutions to be the domain of the corresponding operator. In this paper we will always refer constructed physical observables as Dirac observables even though they do not act on the full physical solutions space.

The motivation for the construction of the Hubble operator comes from the fact that Wheeler-DeWitt equation of minisuperspace quantum cosmology for the FRW-deSitter universe can be written as an eigenvalue equation of an operator which is classically proportional to the square of the Hubble parameter. Importantly this operator involves the inverse power of the scale factor. In isotropic loop quantum cosmology there exists well defined, bounded operators for the inverse powers of scale factor and they are constructed using the techniques which are used in the quantization of the full theory [11. These operators have completely different spectrum compared to the classical values near the classical singularity. So as one expects, we find that the expectation value of the Hubble operator constructed for the spatially flat isotropic loop quantum cosmology behaves in a completely different way for a period of evolution near the classical singularity and this period can be interpreted as a phase of super-inflation.

In the section 2 of this paper, we review the must have properties of the physical observables in the context of both the classical theory and the quantum theory. In section 3, we consider the classical flat FRW universe. In the next section (section 4) we recall [12 the Hamiltonian of the flat FRW universe in terms of Ashtekar connection and densitized triad and then perform minisuperspace quantization. In the same section we give a construction for the Hubble operator as a Dirac observable of this model.

In the beginning of the section 5, we briefly review the kinematics of the isotropic loop quantum cosmology. Then we study the properties of the physical solution space and construct a class of localized Dirac observables for the isotropic flat loop quantum cosmology. We use this construction to define the Hubble operator using the expression of the Hubble operator defined in the context of minisuperspace quantization. For evaluating the physical expectation value of the operator we define a physical inner product which is motivated from the reformulation of ordinary quantum mechanics as constrained dynamics. Considering two simple examples we evaluate the expectation value of the Hubble operator explicitly and then discuss its generic properties near the classical singularity.

\section{Dirac Observables and Localization}

To understand the physical consequences of a dynamical theory, it is absolutely necessary to figure out what are the physical observables of the theory. Here we recall some of the key observations by Gaul and Rovelli [10].

Let us consider a classical dynamical system which has gauge symmetry in its basic variables. For such a system, given a set of initial conditions, it is not possible for equation of motion to uniquely specify the evolution of its basic variables. Let us consider two solutions of equation of motion say $\phi(t)$ and $\phi^{\prime}(t)$ which evolve from the same set of initial conditions but get separated at some later time. In other words $\phi\left(t_{0}\right)=\phi^{\prime}\left(t_{0}\right)$ but $\phi(t) \neq \phi^{\prime}(t)$ for $t>t_{0}$ where $t_{0}$ is the initial time. It was argued by Dirac that for such a case $\phi(t)$ and $\phi^{\prime}(t)$ must be regarded as physically indistinguishable. If this is not the case then the classical determinism

principle will fail to be true. 
To avoid such a circumstances, the physical observables of a theory are defined to be only those functions $\mathcal{O}(\phi(t))$ of basic variables which do not distinguish between $\phi(t)$ and $\phi^{\prime}(t)$ i.e. $\mathcal{O}(\phi(t))=\mathcal{O}\left(\phi^{\prime}(t)\right)$. As it was first systematically defined [13] by Dirac they are also called Dirac observables. Thus it is clear that dynamical predictions only about Dirac observables can be physically relevant.

Now one may ask whether all physical quantities that we can measure are necessarily Dirac observables? The answer is no. To clarify this let us consider the example of a simple pendulum. The classical theoretical setup contains a deflection angle $\alpha$, a clock measuring time $t$ and a dynamical law namely the Newton's Law. Given a set of initial conditions, from the dynamical law it is possible to uniquely predict the deflection angle $\alpha$ at any time $t$ namely $\alpha(t)$. So $\alpha(t)$ is a Dirac observable of the theory. However the time $t$ itself cannot be predicted from the theory. This implies that the time $t$, though we can measure it within the same setup, is not a Dirac observable of the theory. Another way to say it is that the time $t$ is a parameter of the theory not a dynamical variable. But surely without the association of time, the deflection angle $\alpha$ alone cannot describe the dynamics of the system.

Following Gaul and Rovelli [10] let us introduce the notion of partial observables (see [14] for more details). For the pendulum system, time $t$ is an independent partial observable and deflection angle $\alpha$ is a dependent partial observable. Partial observables individually are not physically meaningful quantities of the theory unless they are combined to form the complete observables like $\alpha(t)$. So to be physically relevant, a Dirac observable must be represented by a complete observable. Formally Dirac observables of a classical theory with constraints are defined to be only those functions of the phase space variables whose Poisson bracket vanishes atleast weakly with all the first class constraints.

The essential change that is required when we move into Einsteinian framework from Newtonian framework, is the modification in the notion of reference system. In the Newtonian framework there is a fixed space and a fixed time. These objects define the frame of reference for a dynamical system. Most importantly the frame of reference is completely independent of the dynamics of the system. Now the frame of reference defines the independent partial observables whereas dynamical variables of the system define the dependent partial observables. In construction of a complete observable for the system, we need to associate independent partial observables with the dependent partial observable. Following Gaul and Rovelli [10], let us call this process the localization of the dynamical variable in the frame of reference. Only localized objects can be the candidates for the physically relevant Dirac observables of the theory.

In general relativity situations are very different from that of Newtonian framework. In a generally covariant theory, the puzzle of hole argument (see [10] and reference therein) and its resolution imply that it is no longer possible to separate the frame of reference from the dynamics of the system. So in general relativity we can no longer distinguish an independent partial observable from a dependent partial observable. As a consequence the physical observables in general relativity are defined only with respect to each other. In other words for the purpose of localization which is necessary to define a complete observable, we must use some of the degrees of freedom of the theory either from the matter sector or from the gravitational sector.

In the canonical quantization of a classical theory the classical observables are represented by operators on an appropriate Hilbert space. For Dirac observables, the requirement of vanishing Poisson bracket is turned into the requirement of vanishing commutator bracket with all the constraints atleast on the space of physical solutions. 
From a quantum theory one obtains the physically relevant quantities from the expectation values of the operators in the physical states. So it is naturally expected that in a quantum theory of general relativity the Dirac observables and its expectation value should incorporate the notion of classical localization in an appropriate way.

In the case of isotropic loop quantum cosmology we will see that for a class of operators the requirement of vanishing commutator with the Hamiltonian constraint on the physical solution space itself provides a natural prescription for the localization of the operators.

\section{Classical FRW Cosmology}

In general relativity, the dynamics of gravity manifest itself through the dynamics of spacetime. A homogeneous and isotropic spacetime is described by the Friedmann-RobertsonWalker (FRW) metric. For the spatially flat FRW spacetime the invariant line element, in natural units $(c=\hbar=1)$, is given by

$$
d s^{2}=-d t^{2}+a^{2}(t) \mathbf{d} \mathbf{x}^{2},
$$

where $a(t)$ is the scale factor of the FRW universe.

The dynamics of the spacetime (10) is described by the famous Friedmann equation

$$
3\left(\frac{\dot{a}}{a}\right)^{2}=k \rho+\Lambda,
$$

where overdot denotes the derivative with respect to coordinate time $t, k:=8 \pi G$ is the gravitational coupling constant, $\rho$ is the homogeneous energy density of the matter fields and $\Lambda$ is the cosmological constant. Friedmann equation (2) along with covariant conservation of matter stress tensor completely determine the evolution of FRW universe upto the boundary conditions.

We now consider the simplest example of a FRW universe where the dynamics is completely controlled by a non zero cosmological constant. This universe is also known as deSitter universe. The Friedmann equation (2) for the FRW-deSitter case leads to

$$
\mathcal{H}^{2}=\frac{\Lambda}{3}
$$

where the quantity $\mathcal{H}:=\left(\frac{\dot{a}}{a}\right)$ is the Hubble parameter. One should notice here that the Hubble parameter for FRW-deSitter universe is a constant.

Throughout this paper we will consider FRW-deSitter universe as a test case for comparing results of classical cosmology, minisuperspace (Wheeler-DeWitt) quantum cosmology and loop quantum cosmology.

\section{Minisuperspace (Wheeler-DeWitt) Quantum Cosmology}

In minisuperspace quantization [15, 16] of gravity, at first one reduces the classical phase space of general relativity by the symmetry of the spacetime. Thereafter one uses the symmetry reduced phase space variables for the quantization procedure.

The Einstein-Hilbert action for the spatially flat FRW spacetime (1) can be written as

$$
S_{\text {grav }}:=\int d t L_{\text {grav }}
$$


where

$$
L_{\text {grav }}=-\frac{3 a \dot{a}^{2}}{k}-\frac{\Lambda}{k} a^{3} .
$$

In getting expression(15) for $L_{\text {grav }}$ we have dropped a total derivative term. Moreover to avoid infinity due to integration over whole space, as integrand is independent of position, we have compactified the space and taken the coordinate volume integral to be one.

The spatial part of the FRW metric is given by $g_{a b}=a^{2} \delta_{a b}$. So the metric has just one degree of freedom. For simplicity we now define a new position variable $Q:=a^{2}$. The corresponding conjugate momentum is given by $P:=\frac{\partial L_{\text {grav }}}{\partial \dot{Q}}$. Then the Hamiltonian for the spacetime is given by

$$
H_{\text {grav }}:=P \dot{Q}-L_{\text {grav }}=-\frac{k}{3} P^{2} \sqrt{Q}+\frac{\Lambda}{k} Q^{\frac{3}{2}} .
$$

Now $Q$ and $P$ being a canonical pair, we can perform minisuperspace quantization [15, 16] using them. However as we intend to import few ideas into loop quantum cosmology, we define a new set of canonical pair as

$$
\begin{aligned}
c & :=\gamma \dot{a}=-\frac{1}{3} \gamma k P, \\
p & :=a^{2}=Q
\end{aligned}
$$

where $\gamma$ is a positive real number. In the context of loop quantum gravity $\gamma$ is known as Barbero-Immirzi parameter [17, 18] and the new canonical pair $c$ and $p$ are the Ashtekar connection and the densitized triad for the homogeneous and isotropic spacetime. It is easy to check that the Poisson bracket between these new variables is given by

$$
\{c, p\}=\frac{1}{3} \gamma k \text {. }
$$

Including the matter sector and using the new pair of variables $(c, p)$ the full Hamiltonian can be written as

$$
H:=H_{\text {grav }}+H_{\text {matter }}=-\frac{3}{k \gamma^{2}} c^{2} \sqrt{p}+\frac{\Lambda}{k} p^{\frac{3}{2}}+H_{\text {matter }} .
$$

In performing canonical quantization, one represent the classical phase space variables as operators on an appropriate Hilbert space and their classical Poisson bracket is turned into a commuter bracket for the operators. Here it is convenient to choose triad representation for the canonical quantization. In this representation triad acts as a multiplication operator whereas the connection acts as a derivative operator which is given by

$$
\hat{c}=\frac{i}{3} \gamma k \frac{d}{d p} .
$$

Since $\hat{c}, \hat{p}$ do not commute with each other, the ordering ambiguity arises naturally in constructing the Hamiltonian operator. Here we will choose the connection to the left ordering as this appears as an approximation of Wheeler-DeWitt operator [19] of isotropic loop quantum cosmology [20]. 
In the quantum theory the Hamiltonian acts as a constraint operator on the kinematical Hilbert space, because in the classical general relativity the corresponding Hamiltonian vanishes. The physical states $\psi(p)$ of the quantum theory are selected out by the requirement that the Hamiltonian constraint (10) annihilate the physical states i.e.

$$
\hat{H} \psi(p)=0 .
$$

To solve the equation (12) explicitly we need to specify the matter sector Hamiltonian $H_{\text {matter }}$. Here again we consider the FRW-deSitter universe. For this case the matter sector Hamiltonian is zero. Using the expression for derivative operator $\hat{c}$, the Hamiltonian constraint leads to a second order linear differential equation which is given by

$$
\frac{d^{2}}{d p^{2}}(\sqrt{p} \psi(p))+b^{2} p^{\frac{3}{2}} \psi(p)=0
$$

where $b^{2}=\frac{3 \Lambda}{k^{2}}$. The solutions of the equation (13) are given by Bessel functions of fractional order $\frac{1}{3}$ and $-\frac{1}{3}$ i.e.

$$
\psi_{ \pm}(p)=J_{ \pm \frac{1}{3}}\left(\frac{2}{3} b p^{\frac{3}{2}}\right)
$$

Physical observables of a theory are represented by Dirac observables. In the quantum theory it amounts to the requirement that the corresponding operator $\hat{O}$ must commute with the Hamiltonian constraint at least on the space of physical states i.e.

$$
[\hat{O}, \hat{H}] \psi(p)=0
$$

From the equation (13), we observe that the $\psi_{ \pm}(p)$ appear as the "eigen functions" of the operator $p^{-\frac{3}{2}} \frac{d^{2}}{d p^{2}} \sqrt{p}$. This operator classically corresponds to $-\frac{9}{\gamma^{2} k^{2}} \frac{c^{2}}{p}=-\frac{9}{k^{2}}\left(\frac{\dot{a}}{a}\right)^{2}$. Most importantly the classical quantity is proportional to the square of the Hubble parameter. Observing this fact, we can now define the formal Hubble operator for this model as

$$
\hat{\mathcal{H}}^{2}=-\frac{k^{2}}{9} p^{-\frac{3}{2}} \frac{d^{2}}{d p^{2}} \sqrt{p} .
$$

$\psi_{ \pm}(p)$ being eigen functions, $\hat{\mathcal{H}}^{2}$ trivially satisfy (15) and so $\hat{\mathcal{H}}^{2}$ is a Dirac observable. It is possible to choose a different operator ordering other than (16) such that the new operator also corresponds classically to the square of Hubble parameter. However all such operators fail to commute with the Hamiltonian constraint on the physical solutions space. The formal expectation value of this operator in the physical states, with respect to the kinematical inner product, is given by

$$
\left\langle\hat{\mathcal{H}}^{2}\right\rangle:=\frac{\left\langle\psi_{ \pm}(p), \hat{\mathcal{H}}^{2} \psi_{ \pm}(p)\right\rangle}{\left\langle\psi_{ \pm}(p), \psi_{ \pm}(p)\right\rangle}=\frac{\Lambda}{3} .
$$

So we find that the expectation value of the Hubble operator $\hat{\mathcal{H}}^{2}$ is exactly equal to the classical value (3). In other words, in the example considered here, the minisuperspace quantization does not give any correction to classical Friedmann equation (3). However it is important to emphasize here that, for a general constrained system, the definition of expectation value (17) is not always a well defined quantity as the solutions for such system are not always square-integrable functions. 


\section{$5 \quad$ Isotropic Loop Quantum Cosmology}

The isotropic loop quantum cosmology [20] is defined to be the sector of full loop quantum gravity whose kinematical [3] distributional states are supported only on homogeneous and isotropic connections. Unlike minisuperspace quantization, in loop quantum cosmology symmetry reduction are done at the quantum level. So many features of the full theory like discrete volume spectrum [21] survive the symmetry reduction.

\subsection{Kinematics and Dynamics}

In this paper we will consider only spatially flat model. We will follow mainly the conventions of [22, 23]. The symmetry reduced kinematical Hilbert space for the isotropic loop quantum cosmology is given by $L^{2}\left(S U(2), d \mu_{H}\right)$. An orthonormal basis set for the kinematical Hilbert space is given by $\{|n\rangle \mid n \in \mathbb{Z}\}$. These basis states are the eigenstates of the isotropic triad operator. Here we define a new triad operator $\hat{p}$ whose eigenvalues differ from that of [23] by a factor of $l_{p}^{2}$. The action of this operator $\hat{p}$ on the triad basis states is defined as

$$
\hat{p}|n\rangle=\frac{1}{6} \gamma n|n\rangle .
$$

One should notice here the eigenvalues of $\hat{p}$ are dimensionless. So this operator can be related with the scale factor of the FRW metric (11) directly by the relation(8).

In the connection representation, the functional form of these basis states is given by

$$
\langle c \mid n\rangle=\frac{\exp \left(\frac{1}{2} i n c\right)}{\sqrt{2} \sin \left(\frac{1}{2} c\right)}
$$

The isotropic volume operator and the inverse scale factor operator both are diagonal in these basis. The action of the volume operator on these basis is given by

$$
\hat{V}|n\rangle=\left(\frac{1}{6} \gamma l_{p}^{2}\right)^{\frac{3}{2}} \sqrt{(|n|-1)|n|(|n|+1)}|n\rangle=: V_{\frac{1}{2}(|n|-1)}|n\rangle,
$$

and the action of the inverse scale factor is given by

$$
a^{\hat{-1}}|n\rangle=16 \gamma^{-2} l_{p}^{-3}\left(\sqrt{V_{\frac{1}{2}|n|}}-\sqrt{V_{\frac{1}{2}|n|-1}}\right)^{2}|n\rangle .
$$

Here again we have defined the operator such that the eigenvalues of the inverse scale factor are dimensionless.

A general kinematical state in these basis can be written as

$$
|s\rangle=\sum_{n \in \mathbb{Z}} s_{n}|n\rangle,
$$

where coefficients $s_{n}$ 's are in fact vectors of the Hilbert space corresponding to the matter sector. In coordinate representation of conventional quantization of matter sector, $s_{n}$ 's are complex valued functions of the matter degrees of freedom.

The full Hamiltonian constraint of loop quantum cosmology consists of gravitational sector as well as matter sector. In standard dynamical system, gravity couples with matter sector via metric components. In loop quantum gravity metric variables are replaced by triads. So 
complete geometrical properties are encoded in the triad components whereas its dynamics is encoded in the connection components. Thus for standard gravity-matter coupling, the matter sector Hamiltonian is diagonal in the triad basis. The action of gravitational Hamiltonian on the triad basis is given by

$$
\hat{H}_{\text {grav }}|n\rangle=\frac{3}{2} \gamma^{-2}\left(k \gamma l_{p}^{2}\right)^{-1} \operatorname{sgn}(n)\left(V_{\frac{1}{2}|n|}-V_{\frac{1}{2}|n|-1}\right)(|n+4\rangle-2|n\rangle+|n-4\rangle),
$$

and the action of matter Hamiltonian is given by

$$
\hat{H}_{\text {matter }}|n\rangle=H_{\text {matter }}(n)|n\rangle .
$$

In (24), $H_{\text {matter }}(n)$ is just the symbolic eigenvalue of the matter Hamiltonian but one should remember that it is still an operator on the Hilbert space of matter sector.

To select out the physical states $|s\rangle$ one imposes Hamiltonian constraint as

$$
\hat{H}|s\rangle:=\left(\hat{H}_{\text {grav }}+\hat{H}_{\text {matter }}\right)|s\rangle=0 .
$$

The combined action of $\hat{H}_{\text {grav }}$ and $\hat{H}_{\text {matter }}$ on the state $|s\rangle$ leads to

$$
\sum_{n \in \mathbb{Z}}\left(A_{n+4} s_{n+4}+B_{n} s_{n}+A_{n-4} s_{n-4}\right)|n\rangle=0
$$

where $A_{n}=V_{\frac{1}{2}|n|}-V_{\frac{1}{2}|n|-1}$ and $B_{n}=-2 A_{n}+\frac{2}{3} \gamma^{2}\left(k \gamma l_{p}^{2}\right) H_{\text {matter }}(n)$. In (26) we have absorbed $\operatorname{sgn}(n)$ in the definition of $s_{n}$. Now the equation (26) requires coefficient of all $|n\rangle$ to vanish. The coefficient of $|n+4\rangle$ leads to the difference equation

$$
A_{n+8} s_{n+8}+B_{n+4} s_{n+4}+A_{n} s_{n}=0 .
$$

The difference equation(27) for $s_{n}$ can also be regarded as an evolution equation [24] with respect to an internal time. There one chooses triad basis index $n$ as an internal time. $n$ being discrete, the evolution is necessarily discrete evolution.

\subsection{Physical Solution Space}

The equation (27) is an eighth order difference equation but with equal steps of four. This implies that we can divide all solution $s_{n}$ 's of (27) into four sector and each sector can be completely determined by specifying two initial conditions.

We now define the set of all $s_{n}$ which are solution of (27) with the given initial conditions $\left(s_{i}, s_{4+i}\right)$ as

$$
G_{i}\left(s_{i}, s_{4+i}\right):=\left\{s_{4 n+i} \mid n \in \mathbb{Z}\right\},
$$

where $i=0,1,2,3$. The state vector corresponding to the set $G_{i}\left(s_{i}, s_{4+i}\right)$ is given by

$$
\left|G_{i}\right\rangle_{\left(s_{i}, s_{4+i}\right)}:=\sum_{n} s_{4 n+i}|4 n+i\rangle
$$

So clearly any physical state $|s\rangle$ can be written as

$$
|s\rangle=\left|G_{0}\right\rangle_{\left(s_{0}, s_{4}\right)}+\left|G_{1}\right\rangle_{\left(s_{1}, s_{5}\right)}+\left|G_{2}\right\rangle_{\left(s_{2}, s_{6}\right)}+\left|G_{3}\right\rangle_{\left(s_{3}, s_{7}\right)} .
$$


The state $\left|G_{i}\right\rangle_{\left(s_{i}, s_{4+i}\right)}$ 's are orthogonal to each other. Moreover by construction the Hamiltonian constraint will annihilate them individually. Thus $\left|G_{i}\right\rangle_{\left(s_{i}, s_{4+i}\right)}$ 's are also physical states.

To specify a general solution of (27) one requires to supply eight initial conditions. But we have seen that we can decompose a general solution into four orthogonal solutions each of which requires just two initial conditions and they cannot be decomposed further. We will see in the next section that this splitting is crucial for the construction of a class of Dirac observables of isotropic loop quantum cosmology. Moreover for the following discussion it is sufficient to consider just one such sector say $G_{1}:=\left\{\left|G_{1}\right\rangle_{\left(s_{1}, s_{5}\right)}\right\}$. Clearly any physical state in this sector can be uniquely constructed by specifying values of $s_{1}$ and $s_{5}$.

If we now consider the $s_{1}-s_{5}$ plane (see fig. 1), corresponding to every point in the plane there exist a physical state in the $G_{1}$ sector.

\subsection{Localized Dirac Observable}

Any function of classical phase space variables of isotropic loop quantum cosmology can be either purely a function of triad or purely a function of connection or it could be a function of both. For simplicity, we will consider here only the functions of the first category. The corresponding operators are diagonal in the triad basis. We have already argued that for a dynamical system, the physically relevant Dirac observables must be localized in an appropriate frame of reference. In the following construction for the localized Dirac observable, we start with a kinematical operator and then explore the requirement on physical solution space such that the action of the kinematical operator commutes with the Hamiltonian constraint at some fixed time slice. Using this requirement we show that it is not possible for a single Dirac observable to represent the action of the kinematical operator on all the triad basis vectors. Then we give a procedure for construction of Dirac observables for a given kinematical operator. Although we start with a kinematical operator whose classical expression can be written as a pure function of triad variable, at the end of the construction, the corresponding Dirac observables do not have this property i.e. they cannot be classically represented by simple analytic functions of triad variable.

The action of an diagonal operator say $\hat{O}$, on the triad basis can be written as

$$
\hat{O}|n\rangle=f_{n}|n\rangle \text {. }
$$

We now consider a general state $\left|s^{\prime}\right\rangle$ which is spanned by the basis set $\{|4 n+1\rangle \mid n \in \mathbb{Z}\}$ as

$$
\left|s^{\prime}\right\rangle=\sum_{m} s_{m}^{\prime}|m\rangle
$$

where $m=4 n+1$. The action of the operator $\hat{O}$ on the state $\left|s^{\prime}\right\rangle$ is given by

$$
\hat{O}\left|s^{\prime}\right\rangle=\sum_{m} s_{m}^{\prime} f_{m}|m\rangle \text {. }
$$

The projection of the action of the Hamiltonian on the state $\left|s^{\prime}\right\rangle$ at a fixed "time slice" say $\left(m_{0}+4\right)$, is given by

$$
\left\langle m_{0}+4|\hat{H}| s^{\prime}\right\rangle=A_{m_{0}+8} s_{m_{0}+8}^{\prime}+B_{m_{0}+4} s_{m_{0}+4}^{\prime}+A_{m_{0}} s_{m_{0}}^{\prime} .
$$

If the state $\left|s^{\prime}\right\rangle$ also belongs to the physical solution space then the coefficients $s_{m_{0}+8}^{\prime}$, $s_{m_{0}+4}^{\prime}$ and $s_{m_{0}}^{\prime}$ will satisfy

$$
A_{m_{0}+8} s_{m_{0}+8}^{\prime}+B_{m_{0}+4} s_{m_{0}+4}^{\prime}+A_{m_{0}} s_{m_{0}}^{\prime}=0 .
$$


$m_{0}$ being a fixed time slice, the equation (35) is just a relation and not the difference equation.

Similarly the projection of the action of the Hamiltonian on the state $\hat{O}\left|s^{\prime}\right\rangle$ at the same time slice $\left(m_{0}+4\right)$, is given by

$$
\left\langle m_{0}+4|\hat{H} \hat{O}| s^{\prime}\right\rangle=A_{m_{0}+8} s_{m_{0}+8}^{\prime \prime}+B_{m_{0}+4} s_{m_{0}+4}^{\prime \prime}+A_{m_{0}} s_{m_{0}}^{\prime \prime},
$$

where $s_{m_{0}+8}^{\prime \prime}=f_{m_{0}+8} s_{m_{0}+8}^{\prime}, s_{m_{0}+4}^{\prime \prime}=f_{m_{0}+4} s_{m_{0}+4}^{\prime}$ and $s_{m_{0}}^{\prime \prime}=f_{m_{0}} s_{m_{0}}^{\prime}$. We now explore the requirement on $\left(s_{m_{0}+8}^{\prime}, s_{m_{0}+4}^{\prime}, s_{m_{0}}^{\prime}\right)$ such that $\left(s_{m_{0}+8}^{\prime \prime}, s_{m_{0}+4}^{\prime \prime}, s_{m_{0}}^{\prime \prime}\right)$ also satisfy the same relation (35) i.e.

$$
A_{m_{0}+8} s_{m_{0}+8}^{\prime \prime}+B_{m_{0}+4} s_{m_{0}+4}^{\prime \prime}+A_{m_{0}} s_{m_{0}}^{\prime \prime}=0 \text {. }
$$

The equations (35) and (37) together lead to a consistency requirement on $s_{m_{0}+4}^{\prime}$ and $s_{m_{0}}^{\prime}$ and that is given by

$$
\left(f_{m_{0}+8}-f_{m_{0}+4}\right) B_{m_{0}+4} s_{m_{0}+4}^{\prime}+\left(f_{m_{0}+8}-f_{m_{0}}\right) A_{m_{0}} s_{m_{0}}^{\prime}=0 .
$$

For the trivial case where the functions $f_{m_{0}}$ 's are independent of $m_{0}$ the equation (38) is empty. We can now interpret the relation (37) also as a projection of the action of Hamiltonian on some physical state $\left|s^{\prime \prime}\right\rangle$ at the same time slice $\left(m_{0}+4\right)$.

$\left|s^{\prime}\right\rangle$ being a physical state, by specifying the values of $s_{m_{0}+4}^{\prime}$ and $s_{m_{0}}^{\prime}$, we can construct all other $s_{m}^{\prime}$ using the difference equation (27). Since the equation (27) is a linear difference equation then the consistency requirement (38) will lead to a linear equation between $s_{1}^{\prime}$ and $s_{5}^{\prime}$. We write this linear equation as

$$
s_{5}^{\prime}=\beta^{\prime}\left(m_{0}+4\right) s_{1}^{\prime} .
$$

Similarly starting from $s_{m_{0}+4}^{\prime \prime}$ and $s_{m_{0}}^{\prime \prime}$ one can construct the whole tower of $s_{m}^{\prime \prime}$ using the difference equation (27). So by construction $\left|s^{\prime \prime}\right\rangle$ will be a physical state. We denote the relation between $s_{1}^{\prime \prime}$ and $s_{5}^{\prime \prime}$ for $\left|s^{\prime \prime}\right\rangle$ as

$$
s_{5}^{\prime \prime}=\beta^{\prime \prime}\left(m_{0}+4\right) s_{1}^{\prime \prime} .
$$

In $s_{1}-s_{5}$ plane (see fig. 1) two physical states $\left|s^{\prime}\right\rangle$ and $\left|s^{\prime \prime}\right\rangle$ are described by a point on the straight lines of different slope. We now define a set of physical states of the $G_{1}$ sector as $D_{m_{0}+4}:=\left\{\left|G_{1}\right\rangle_{\left(s_{1}, s_{5}\right)} \mid s_{5}=\beta^{\prime}\left(m_{0}+4\right) s_{1}\right\}$.

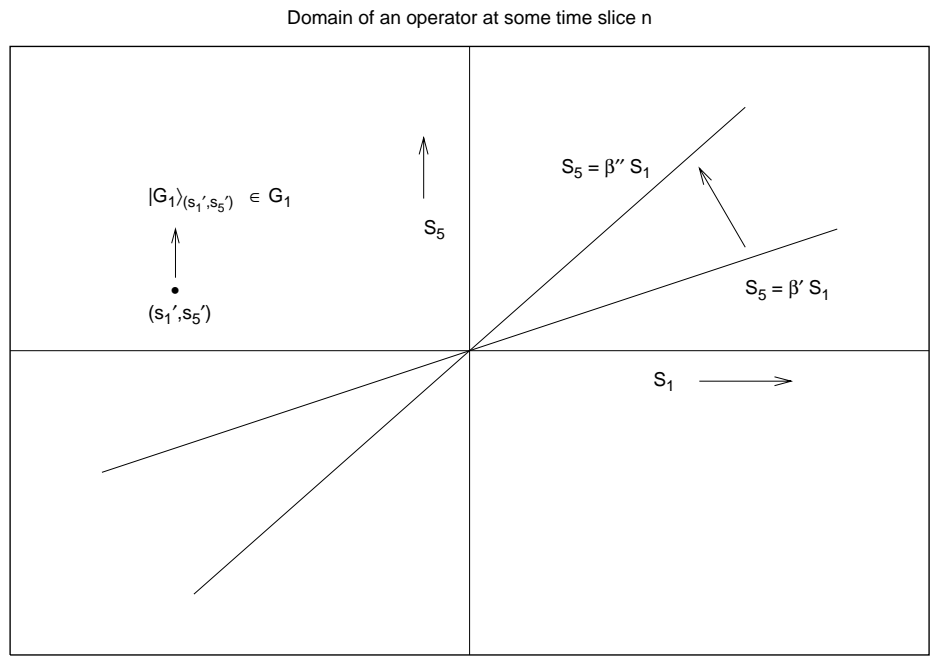

Figure 1. Domain of an operator at some time slice n. 
Similar argument readily goes through for the $G_{2}$ and $G_{3}$ sector. But for $G_{0}$ sector equation (38) leads to $s_{4}=0$. Consequently all $s_{4 n}$ drop out except for $s_{0}$ which anyway cannot be determined by (27) as it decouples from the rest of $s_{n}$ 's. We now generalize the definition of the subspaces as $D_{4 n+i}:=\left\{\left|G_{i}\right\rangle_{\left(s_{i}, s_{4+i}\right)} \mid s_{4+i}=\beta^{\prime}(4 n+i) s_{i}\right\}$. For the $G_{0}$ sector the subspaces are trivial and they are given by $D_{4 n}=\left\{\left|G_{0}\right\rangle_{\left(s_{0}, s_{4}\right)}=s_{0}|0\rangle\right\}$. One may note here that the subspaces exclude the pre-classical solutions.

For the operator $\hat{O}$ (31) to be a Dirac observable one requires it to satisfy

$$
\hat{H} \hat{O}|s\rangle=0
$$

where $|s\rangle$ is a physical state. We use the decomposition (30) of the physical state $|s\rangle$. Then the equation (41) implies $\hat{H} \hat{O}\left|G_{i}\right\rangle_{\left(s_{i}, s_{4+i}\right)}=0$, for all $i$. Here we consider just the $G_{1}$ sector of the physical solution space. Inserting the complete set of basis from the left, we get the requirement in the $G_{1}$ sector as

$$
\sum_{n}|n\rangle\left\langle n|\hat{H} \hat{O}| G_{1}\right\rangle_{\left(s_{1}, s_{5}\right)}=\sum_{m}|m\rangle\left\langle m|\hat{H} \hat{O}| G_{1}\right\rangle_{\left(s_{1}, s_{5}\right)}=0
$$

where $m=4 n+1$.

The equation (42) requires the coefficient $\left\langle m|\hat{H} \hat{O}| G_{1}\right\rangle_{\left(s_{1}, s_{5}\right)}$ to vanish for all time slice $m$. We have seen that this requirement, at a given time slice $m$, can be satisfied provided the operator $\hat{O}$ acts only on a subspace $D_{m}$ of the $G_{1}$ sector of the physical solution space. To ensure this we need to define the domain of $\hat{O}$ in $G_{1}$ sector to be the subspace $D_{m}$. Now the domain $D_{m}$ being $m$ dependent, we must tag the operator $\hat{O}$ with $m$ as well. This implies that to satisfy (42) for all $m$ we need to have a collection of operators each tagged with $m$.

We now define the tagged operator $\hat{O}_{m}$ as

$$
\left|s^{\prime \prime}\right\rangle=\hat{O}_{m}\left|s^{\prime}\right\rangle
$$

where $\left|s^{\prime}\right\rangle \in D_{m}$ and $\left|s^{\prime \prime}\right\rangle$ is constructed using the difference equation (27) with the given values of $s_{m}^{\prime \prime}=f_{m} s_{m}^{\prime}, s_{m-4}^{\prime \prime}=f_{m-4} s_{m-4}^{\prime}$ and the operator $\hat{O}_{m}$ is assumed to be diagonal like the kinematical operator $\hat{O}$. Using the definition (43), one can uniquely construct the matrix elements of $\hat{O}_{m}$ in the triad basis of $G_{1}$ sector and this is possible only because of the fact that the physical states split into four orthogonal states; each requiring just two initial conditions. This operator $\hat{O}_{m}$ has exactly the same action, as of the kinematical operator $\hat{O}$ (31), on the three successive basis $|m-4\rangle,|m\rangle$ and $|m+4\rangle$ of the $G_{1}$ sector. Now we need to specify the matrix elements of $\hat{O}_{m}$ in $G_{2}, G_{3}$ and $G_{0}$ sectors. Since we have defined the operator $\hat{O}_{m}$ with respect to its nontrivial action on $G_{1}$ sector, we may define the action of the operator $\hat{O}_{m}$ to be trivial on the other sectors. For the trivial action of the operator $\hat{O}_{m}$ on these sectors the corresponding matrix elements of the operator $\hat{O}_{m}$ can be set to zero. With this definition, the domain of a general operator $\hat{O}_{4 n+i}$ in the full physical solution space is given by

$$
\bar{D}_{4 n+i}:=\bigcup_{j \neq i} G_{j} \bigcup D_{4 n+i}
$$

We next consider the situation where the operator $\hat{O}_{m}$ acts non-trivially on $G_{2}, G_{3}$ and $G_{0}$ sectors as well. In the case of $G_{1}$ sector, we have seen that we can set three successive matrix elements of $\hat{O}_{m}$, equal to that of the kinematical operator $\hat{O}$. There we have used the index of middle element to designate the operator itself. However the selection of three 
successive matrix elements of the operator $\hat{O}_{m}$ in the $G_{2}$ and $G_{3}$ sectors can be done various ways. So the domain of the operator $\hat{O}_{m}$, in these two sectors, will depend on the particular choice one makes.

A natural suggestion is to select these elements such that the operator $\hat{O}_{m}$ has maximum number of successive elements that are equal to that of the kinematical operator $\hat{O}$ around the $m$-th element. This suggestion leads to the domain of the operator $\hat{O}_{m}$ in the $G_{2}$ sector to be $D_{m+1}$ and in the $G_{3}$ sector the domain could either be $D_{m-2}$ or $D_{m+2}$.

For the $G_{0}$ sector the subspace $D_{m-1}\left(=D_{4 n}\right)$ is trivial. So in this sector, we can set the matrix elements of the operator $\hat{O}_{m}$ as exactly same as that of the kinematical operator $\hat{O}$. Thus we have specified all the matrix elements of the operator $\hat{O}_{m}\left(=\hat{O}_{4 n+1}\right)$ in the full physical solution space. Following the similar steps one can construct the operators $\hat{O}_{4 n+2}$, $\hat{O}_{4 n+3}$ and $\hat{O}_{4 n}$. With this prescription, in the full physical solution space, the domain of a general operator $\hat{O}_{n}$ can either be chosen to be

$$
\bar{D}_{n}:=D_{n-1} \bigcup D_{n} \bigcup D_{n+1} \bigcup D_{n+2}
$$

or

$$
\bar{D}_{n}:=D_{n-2} \bigcup D_{n-1} \bigcup D_{n} \bigcup D_{n+1}
$$

For any of the above choices, the operator $\hat{O}_{n}$ has the same action, as that of the kinematical operator $\hat{O}$, on at least eleven successive basis states $|n-5\rangle, \ldots|n\rangle, \ldots|n+5\rangle$. The main difference between the two operators $\hat{O}_{n}$ and $\hat{O}$, is that the action of $\hat{O}_{n}$ on a physical state leads to another physical state whereas the action of $\hat{O}$ does not. However they have the same action on the basis state $|n\rangle$ and the neighboring basis states of $|n\rangle$.

Our main aim in this exercise is to construct the Dirac observable corresponding to the given kinematical operator $\hat{O}$ (31). In doing this, we have seen that it is not possible for a single Dirac observable to represent the same action of the kinematical operator $\hat{O}$ on all the triad basis elements. Rather we need to have a set of Dirac observables like $\hat{O}_{n}$. Each Dirac observable $\hat{O}_{n}$ mimics the action of the operator $\hat{O}$ only on and around the basis state $|n\rangle$ but not globally.

In the isotropic loop quantum cosmology, the triad basis index $n$ is interpreted as the internal time index. In other words, these discrete indices define the frame of reference for the evolution of the universe. Naturally the tagging of an operator by the triad index $n$ is an equivalent description of a kind of localization of the operator. In the section 2, we have argued that a Dirac observable to be physically relevant, it must be localized in an appropriate frame of reference. Here, in the case of isotropic loop quantum cosmology, we find a natural prescription for the localization of the Dirac observables of the theory.

We may define a collection of such operators $\hat{O}_{n}$ (associated with the same kinematical operator $\hat{O})$ as

$$
\hat{\mathcal{O}}:=\left\{\hat{O}_{n} ; D\left(\hat{O}_{n}\right):=\bar{D}_{n} \mid n \in \mathbb{Z}\right\} .
$$

The action of any operator of the collection (47) on a physical state of its domain leads to a physical state. For notational simplification we will refer this collection itself as an operator. So symbolically its action can be given as $\left|s^{\prime}\right\rangle=\hat{\mathcal{O}}|s\rangle$ where $|s\rangle,\left|s^{\prime}\right\rangle$ both are physical states. But important point to remember here is that $\hat{\mathcal{O}}$ is not a single operator, rather it is a collection of operators.

We now consider the example of volume operator in the isotropic loop quantum cosmology. This operator is diagonal in the triad basis. The action of the kinematical volume operator $\hat{V}$ 
(20) on a physical state does not lead to a physical state. So the kinematical volume operator is not a Dirac observable. So it is necessary to construct a physical volume operator to study its physical consequences.

Let us think of a situation where it is possible to construct a single volume operator whose action on a physical state leads to another physical state. But without a further prescription of localization, this volume operator is just a partial observable. Hence this operator itself is not a physically relevant object. The total volume of a dynamical universe is a evolving quantity. So it is not enough just to say the amount of total volume, it is also necessary to specify the corresponding time. In other words, the physical volume of the universe must be represented by a complete observable.

Hence the physical volume operator should not only act invariantly on physical solution space but it should also be localized in an appropriate frame of reference. The localized volume operator $\hat{V}_{n}$, constructed using the prescription presented here, precisely does that. So the operator $\hat{V}_{n}$ is a complete observable hence it is a viable candidate for the physical volume operator which represent the total volume of the universe at a given time slice $n$. In the case of isotropic loop quantum cosmology, the localization of the physical volume operator $\hat{V}_{n}$ is not just a choice rather a requirement of the construction.

An important difference between the kinematical volume operator $\hat{V}$ and the localized volume operator $\hat{V}_{n}$ is that the $\hat{V}_{n}$ acts only on a subspace of the physical solution space whereas $\hat{V}$ can act on the entire physical solution space but its action takes physical states outside the physical solution space. However the operators $\hat{V}$ and $\hat{V}_{n}$ act identically on the basis state $|n\rangle$ and its neighboring basis states.

\subsection{Physical Inner Product}

Although there is a well defined inner product on kinematical Hilbert space, loop quantum cosmology so far does not have an inner product defined on physical solution space. But to obtain physically relevant quantities from a quantum theory, one must have some notion of physical inner product. So we need to define a physical inner product for evaluation of the expectation values of the physical observables.

In this regard, we will try to learn from the reformulation of ordinary quantum mechanics as a constrained dynamics. This approach is motivated by the fact that the Hamiltonian constraint for loop quantum cosmology can be viewed as an evolution equation [24] with respect to an internal time. The basic idea of frozen time description ( see 25] for a brief account and references therein for details) of ordinary quantum mechanics is to extend the classical phase space by two extra dimensions of time $t$ and its canonical conjugate $\pi_{t}$. So time $t$ is no longer a parameter of the theory but a dynamical variable. The extended phase space is given by $\Gamma_{k i n}:=\mathbb{R}^{2} \otimes \Gamma_{0}$ where $\Gamma_{0}$ is the usual phase space. Consequently the kinematical Hilbert space can be given as $\mathcal{H}_{\text {kin }}:=L^{2}(\mathbb{R}, d t) \otimes \mathcal{H}_{0}$ where $\mathcal{H}_{0}$ is the kinematical Hilbert space of ordinary quantum mechanics. So a general state vector in $\mathcal{H}_{k i n}$ can be written as

$$
|\psi\rangle=\int d t|t\rangle \otimes\left|\phi_{t}\right\rangle
$$

where $\left|\phi_{t}\right\rangle$ belongs to $\mathcal{H}_{0}$. In this scheme one performs canonical quantization of this new pair $\left(t, \pi_{t}\right)$ as of an ordinary pair of phase space variables. In ordinary quantum mechanics, physical states satisfy Schrodinger equation. An analogous thing is done in this approach by 
imposing the Hamiltonian constraint on physical states $|\psi\rangle$ as

$$
\left(\hat{\pi}_{t}+\hat{H}(\omega)\right)|\psi\rangle=0
$$

where $\hat{H}(\omega)$ is the Hamiltonian corresponding to $\mathcal{H}_{0}$ and $\omega$ denotes the phase space variables corresponding to $\Gamma_{0}$.

The constraint equation (49) implies that $\left|\phi_{t}\right\rangle$ has to satisfy ordinary Schrodinger equation. The kinematical inner product of two physical states $|\psi\rangle$ and $\left|\psi^{\prime}\right\rangle$ is given by

$$
\left\langle\psi^{\prime} \mid \psi\right\rangle_{k i n}=\int d t\left\langle\phi_{t}^{\prime} \mid \phi_{t}\right\rangle
$$

Given the fact $\left\langle\phi_{t}^{\prime} \mid \phi_{t}\right\rangle$ is independent of time $t,\left\langle\psi^{\prime} \mid \psi\right\rangle_{k i n}$ is not finite. Similar situation arises in loop quantum cosmology when we evaluate the kinematical inner product of two physical states.

In quantum mechanics we know that the physically relevant inner product is given in terms of inner product of $\left|\phi_{t}\right\rangle$ 's. In making the correspondence between the inner products of two different description of same dynamics a natural suggestions is to define a physical inner product of $|\psi\rangle$ 's as

$$
\left\langle\psi^{\prime} \mid \psi\right\rangle_{\text {phys }}(t):=\left\langle\phi_{t}^{\prime} \mid \phi_{t}\right\rangle \text {. }
$$

One should notice that we have defined the physical inner product as a function of time $t$. But given the fact the evolution of $\left|\phi_{t}\right\rangle$ 's are unitary, the definition of physical inner product (51) is in fact independent of time. Our main aim here is to use the same idea in the loop quantum cosmology. When recasted as a first order evolution equation [24] in the internal time, the corresponding evolution matrices in the loop quantum cosmology are not unitary. So we will keep the definition of physical inner product tagged with time. In other words we have defined a family of physical inner products.

In loop quantum cosmology a general state is expressed as

$$
|s\rangle=\sum_{n} s_{n}|n\rangle
$$

where $\{|n\rangle\}$ is the eigen basis of the triad operator. In the interpretation of Hamiltonian constraint as an evolution equation 24], $n$ is taken to be the discrete internal time index. The kinematical inner product of two states $|s\rangle$ and $\left|s^{\prime}\right\rangle$ is given by

$$
\left\langle s^{\prime} \mid s\right\rangle_{k i n}=\sum_{n}\left\langle s^{\prime} \mid n\right\rangle\langle n \mid s\rangle \text {. }
$$

Bringing the analogy with the frozen time description of quantum mechanics we now define a family of physical inner products of two states $|s\rangle$ and $\left|s^{\prime}\right\rangle$, each tagged by the internal time index $n$, as

$$
\left\langle s^{\prime} \mid s\right\rangle_{\text {phys }}(n):=\left\langle s^{\prime} \mid n\right\rangle\langle n \mid s\rangle \text {. }
$$

So the physical expectation value of an operator $\hat{O}$ in the physical state $|s\rangle$, at a given time slice $n$, can naturally be defined as

$$
\langle\hat{O}\rangle_{\text {phys }}(n):=\frac{\langle s \mid \hat{O} s\rangle_{\text {phys }}(n)}{\langle s \mid s\rangle_{\text {phys }}(n)} .
$$




\subsection{Hubble Operator}

In the loop quantum cosmology, the absence of singularity [7] is understood in two ways. Firstly the discrete evolution equation allows the system to evolve through the internal time $n=0$ and secondly there exist well defined, bounded operators for the inverse powers of scale factor [11, 26]. In minisuperspace quantization, however, similar features are clearly absent and thus lead to breakdown of evolution equation and the curvature singularity.

We have seen in the example of minisuperspace quantization that the Hubble operator (16) involves the inverse power of scale factor. So when we move into loop quantum cosmology, it is naturally expected that the corresponding Hubble operator will have completely different behavior near the classical singularity.

The construction presented in the previous section for the localized Dirac observable is valid only for the class of operators which are diagonal in the triad basis. The expression of the Hubble operator (16) contains the connection component in its classical expression. In the isotropic loop quantum cosmology, connections are represented by point holonomies which are not diagonal in the triad basis. So clearly we cannot apply the construction directly. However in the classical expression it is possible to substitute the term $c^{2} \sqrt{p}$ using the equation (10).

Thereafter we can define the Hubble operator for the isotropic loop quantum cosmology as

$$
\hat{\mathcal{H}}^{2}=\frac{k}{3} \hat{p}^{-\frac{3}{2}}\left(\hat{H}_{\text {matter }}+\frac{\Lambda}{k} \hat{p^{\frac{3}{2}}}-\hat{H}\right) .
$$

One should notice that the expression (56) now has the total Hamiltonian term which is obviously not diagonal in the triad basis. But the total Hamiltonian term in (56) effectively drops out whenever the Hubble operator acts on the physical states.

The quantization for the operator $p^{-\frac{3}{2}}$ used in [1] has an adjustable ambiguity parameter. However for illustrative purpose here we will use the simplest possible quantization for $p^{-\frac{3}{2}}$. Using the classical identity $p^{-\frac{3}{2}}=a^{-1} a^{-1} a^{-1}$ we quantize the operator as

$$
\hat{p^{-\frac{3}{2}}}=\hat{a^{\hat{-1}}} \hat{a^{-1}} a^{\hat{-1}} \text {. }
$$

In the case of FRW-deSitter universe the matter sector Hamiltonian is zero. Since the quantity $p^{\frac{3}{2}}$ is proportional to the volume, then using the volume operator $\hat{V}$, we can quantize the operator $p^{\frac{3}{2}}$ as

$$
\hat{p^{\frac{3}{2}}}=l_{p}^{-3} \hat{V} .
$$

We have already pointed out that the total Hamiltonian term in (56) effectively drops out whenever it acts on the physical states. So for evaluating expectation values in the physical states, we just need to consider only the rest of the terms of the kinematical Hubble operator (56). These terms are diagonal in the triad basis.

Naturally, using the method described in the previous section for localized Dirac observables, we obtain the one parameter family of physical Hubble operators $\left\{\hat{\mathcal{H}}_{n}^{2}\right\}$, each labeled by the discrete time index $n$. The localized Hubble operator $\hat{\mathcal{H}}_{n}^{2}$ is a diagonal operator and has the same action that of kinematical Hubble operator(apart from the total Hamiltonian term) on the basis state $|n\rangle$ and its neighboring basis states. 
So for the FRW-deSitter universe the physical expectation value (55) of the Hubble operator is given by

$$
\left\langle\hat{\mathcal{H}}^{2}\right\rangle_{\text {phys }}(n)=\frac{\Lambda}{3}\left(16 \gamma^{-2} l_{p}^{-3}\left(\sqrt{V_{\frac{1}{2}|n|}}-\sqrt{V_{\frac{1}{2}|n|-1}}\right)^{2}\right)^{3} l_{p}^{-3} V_{\frac{1}{2}(|n|-1)} .
$$

For given any operator to be a viable physical observable it must have the correct classical limit. We now show that the Hubble operator constructed here does have the correct classical limit. In this context the classical limit is obtained by taking the large $n$ limit i.e. the large volume limit where one expects the effects of small scale physics are negligible. For the large value of $n$, the expectation value of the Hubble operator is given by

$$
\left\langle\hat{\mathcal{H}}^{2}\right\rangle_{\text {phys }}(n)=\frac{\Lambda}{3}\left(1+\frac{37}{16} \frac{1}{n^{2}}+O\left(\frac{1}{n^{4}}\right)\right) .
$$

In the large volume i.e. in the large $n$ limit $a^{2} \sim \frac{1}{6} \gamma n$. So the correction terms to the classical value in (60) are proportional to the Barbero-Immirzi parameter $\gamma$. In the loop quantum cosmology the discreteness in the spectrums of geometrical operators are controlled by the Barbero-Immirzi parameter $\gamma$. In $\gamma \rightarrow 0$ limit i.e. when the discreteness in the spectrums of geometrical operators disappears then the correction terms in (60) also disappear and the expectation value of the Hubble operator reduces exactly to the classical value like in the case of minisuperspace quantization where the geometrical operators have continuous spectrums. This implies that the quantum correction to the Hubble parameter in the isotropic loop quantum cosmology comes necessarily due to the underlying discrete structure of the space.

In fig. 2, we have plotted the expectation value of the Hubble operator for the FRWdeSitter universe. One should notice that near $n=0$ it has completely different behavior compared to the classical case.

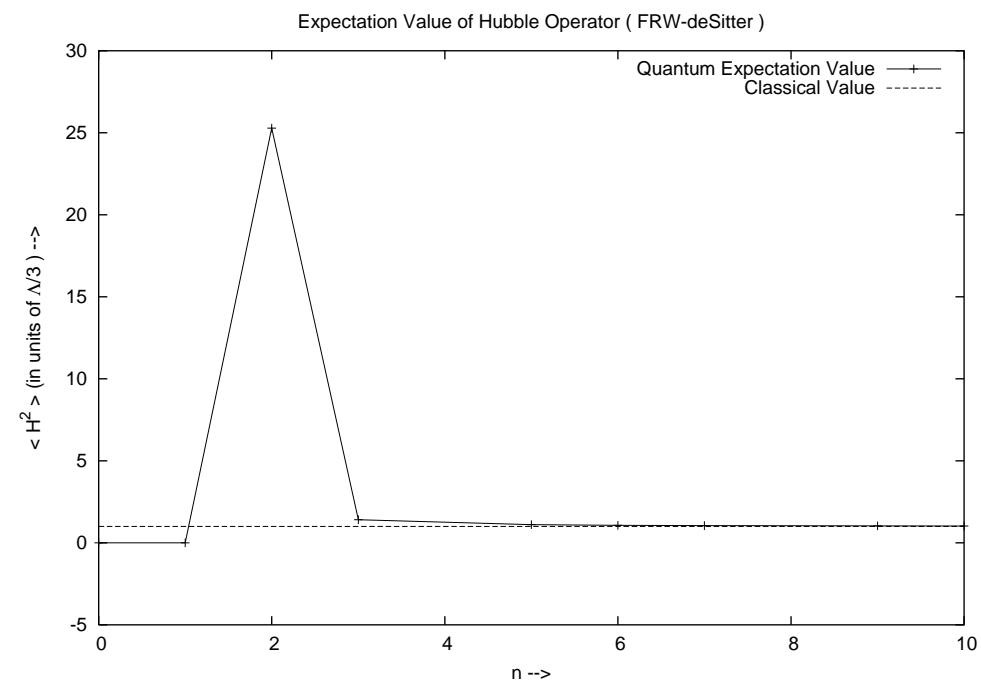

Figure 2. The expectation value of the Hubble operator for the FRW-deSitter universe

We now study the case, considered in [1, of FRW universe with a conventional massless free scalar field. Being free field, the scalar field momentum $p_{\phi}$ is constant say $\omega$. So the corresponding Hamiltonian is given by

$$
H_{\text {matter }}=\frac{1}{2} a^{-3} p_{\phi}^{2}=\frac{1}{2} p^{-\frac{3}{2}} \omega^{2} .
$$


Using the same quantization (57) we obtain the expectation value of the Hubble operator as

$$
\left\langle\hat{\mathcal{H}}^{2}\right\rangle_{\text {phys }}(n)=\frac{k \omega^{2}}{6}\left(16 \gamma^{-2} l_{p}^{-3}\left(\sqrt{V_{\frac{1}{2}|n|}}-\sqrt{V_{\frac{1}{2}|n|-1}}\right)^{2}\right)^{6} .
$$

In fig. 3 we have plotted the expectation value of the Hubble operator for the FRW universe with a conventional massless free scalar field.

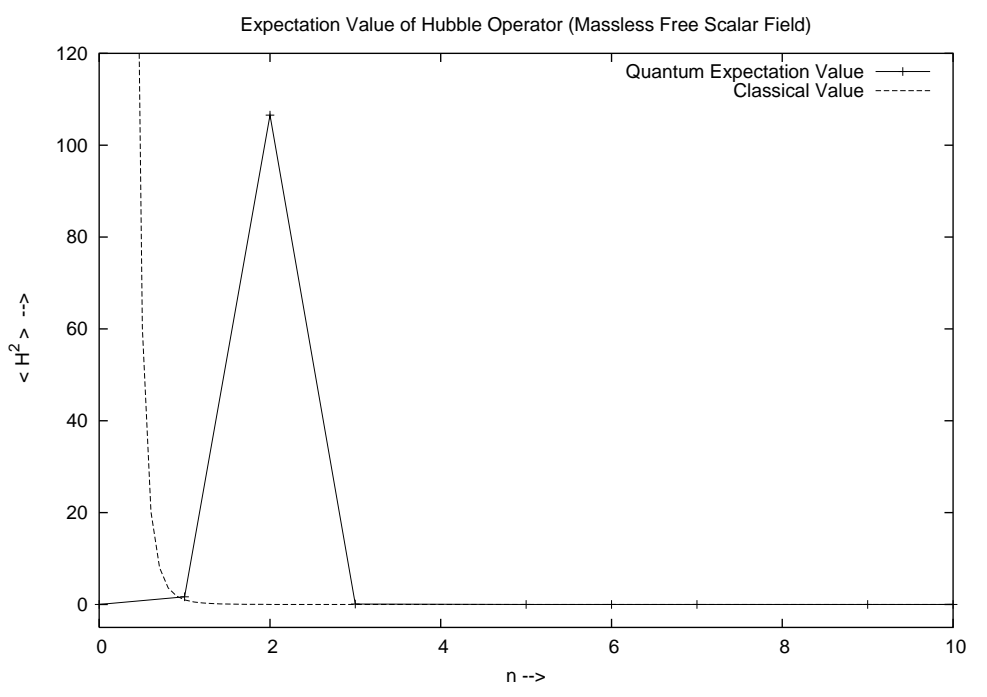

Figure 3. The expectation value of the Hubble operator for a FRW universe with a massless free scalar field

So in both cases we find that there is a period of evolution where the expectation value of the Hubble operator increases with increasing time and then decreases to reach its classical value. Classically increasing Hubble parameter square for expanding universe implies a superaccelerating universe. In the quantum case, the expectation value of the Hubble operator has such behavior. So one may interpret this period as a phase of super-inflation. However, evolution being in discrete steps, one cannot make a direct analogy with the classical case. Also this period exists only for a few steps. But surely the expectation value of the Hubble operator remains larger than the classical value for a period of evolution.

In [1, the phase of super-inflation was obtained from the effective Friedmann equation and the reason for this quantum geometric inflation was cited to be the modification of the kinetic term in the matter Hamiltonian but here we find that apart from the modification of the kinetic term, the existence of $a^{-1}$ term in the Hubble parameter also causes it to behave differently. So this phase seems to be more generic in the loop quantum cosmology. In particular this phase exists even for the FRW-deSitter universe where there is no matter Hamiltonian term. Naturally one gets larger amount of inflation from this picture compared to that from the analysis of effective Friedmann equation. It is important to emphasize here that the consideration of different values for the ambiguity parameter in the quantization of $\hat{p^{-\frac{3}{2}}}$, as used in [1], will lead to further changes in the amount of inflation. 


\section{Conclusions}

To summarize, in this paper we have emphasized the need for localization of Dirac observables of a dynamical theory from a general perspective. In the case of isotropic loop quantum cosmology, we have shown that for a given kinematical operator, one cannot construct the corresponding Dirac observable without localization. Further we have presented a construction by which one can consider a class of operators as localized Dirac observables. These Dirac observables can act only on a subspace of the space of physical solutions. Ideally one would like to have Dirac observables that can act on the full physical solution space. But the construction of Dirac observables here does not allow that. However in the cosmological context it may be more reasonable to have subspaces rather than the full solution space as the domain of Dirac observables, as any "observations" in the cosmology after all would be made with a single state. As an example of these Dirac observables, we have constructed the Hubble operator for the spatially flat isotropic universe.

In the case of closed isotropic model, the same construction of Dirac observable goes through for the diagonal operators. However, using the same substitution as for the case of flat FRW universe, the corresponding Hubble operator cannot be reduced to a function of triads and the total Hamiltonian alone. In this case, the classical expression of the Hubble operator contains an explicit connection term. So to construct the Hubble operator for isotropic closed model, we need to have a procedure for constructing Dirac observables for the functions of connection variables. The corresponding operators are not diagonal in the triad basis. However preliminary investigation in this direction indicates such construction to be viable.

From the physical point of view, specifying a subspace of the physical solution space can also be considered as fixing of some of the initial conditions. We have seen that for a Dirac observable the requirement of vanishing commutator with the Hamiltonian constraint dynamically selects out a subspace of the physical solution space. Thus in this regard, in the isotropic loop quantum cosmology, it seems that Dirac observables themselves supply their initial conditions. As the selections of the subspaces depend on the particular time slices, it also implies that the specification of the initial conditions is dynamical. Moreover this aspect seems to be completely different from that described in [27] where one selects out an unique solution by the requirement of pre-classicality. Furthermore it seems that by combining these two aspects it may be possible to figure out the class of Dirac observables which are relevant in the large volume evolution.

As yet we do not have a definition of physical inner product in the loop quantum cosmology, we have used here a definition of physical inner product which is motivated from the reformulation of ordinary quantum mechanics. But it is important to remember that this definition is a first attempt in this context and need not be the final. In order to define the physical inner product for the states of the frozen time description of the ordinary quantum mechanics, we have seen that it is natural to define a family of physical inner products. However the members of this physical inner products family are not really different from each other as the evolution in ordinary quantum mechanics is unitary. But in the case of isotropic loop quantum cosmology the evolution (when recasted as a first order evolution equation) in the internal time is not unitary. So the physical inner product family members are different from each other but surely they are not completely arbitrary. In this case also the members of the physical inner products family are related to each others by the basic evolution equation as in the case of ordinary quantum mechanics. The expectation values of the Dirac observables of the first category, evaluated with respect to this physical inner product, have zero 
uncertainties. However the physical inner product defined here may not be general enough to be adapted in the full theory so it is desirable that one explores other possible routes as well.

The Hubble parameter is an extremely important quantity of the cosmology. So for the phenomenological purpose it is important to consider more realistic matter Hamiltonian in evaluating the expectation values of the Hubble operator. Apart from that one should also take care of the quantization ambiguities.

\section{Acknowledgements}

I am grateful to Ghanshyam Date for careful reading of the manuscript, further comments and suggestions on it. I am also indebted to him for hours of useful discussions which has helped me to understand many ideas of this subject. I thank Martin Bojowald for providing valuable comments and suggestions on the preprint. I also thank Bobby Ezhuthachan for having productive discussions.

\section{References}

[1] Martin Bojowald, Inflation from Quantum Geometry, Phys.Rev.Lett. 89 (2002) 261301; [arXiv, gr-qc/0206054].

[2] M. Bojowald, H.A. Kastrup, Quantum Symmetry Reduction for Diffeomorphism Invariant Theories of Connections, Class.Quant.Grav. 17 (2000) 3009-3043,[arXiv, hep-th/9907042.

[3] Martin Bojowald, Loop Quantum Cosmology I: Kinematics, Class.Quant.Grav. 17 (2000) 1489-1508; [arXiv, gr-qc/9910103].

[4] Carlo Rovelli, Loop Quantum Gravity, Living Rev.Rel. 1 (1998) 1,[arXiv, gr-qc/9710008.

[5] Thomas Thiemann, Introduction to Modern Canonical Quantum General Relativity, [arXiv, gr-qc/0110034.

[6] Thomas Thiemann, Lectures on Loop Quantum Gravity, [arXiv, gr-qc/0210094.

[7] Martin Bojowald, Absence of Singularity in Loop Quantum Cosmology, Phys.Rev.Lett. 86 (2001) 5227-5230; [arXiv, gr-qc/0102069.

[8] Lee Smolin, Finite, diffeomorphism invariant observables in quantum gravity; Phys.Rev. D 49 (1994) 4028-4040, [arXiv, gr-qc/9302011].

[9] Alejandro Perez, Carlo Rovelli; Observables in quantum gravity, [arXiv, gr-qc/0104034.

[10] Marcus Gaul, Carlo Rovelli, Loop Quantum Gravity and the Meaning of Diffeomorphism Invariance, Lect.Notes Phys. 541 (2000) 277-324; [arXiv, gr-qc/9910079.

[11] Thomas Thiemann, QSD V : Quantum Gravity as the Natural Regulator of Matter Quantum Field Theories, Class.Quant.Grav. 15 (1998) 12811314; [arXiv, gr-qc/9705019]. 
[12] Hideo Kodama, Specialization of Ashtekar's formalism to Bianchi cosmology, Prog. Theor. Phys. 801024 (1988).

[13] P.A.M. Dirac, Lectures on Quantum Mechanics, Belfer graduate school of science, Yeshiva university,1964.

[14] Carlo Rovelli, Partial observables, Phys.Rev. D65 (2002) 124013; [arXiv gr-qc/0110035].

[15] Bryce S. DeWitt, Quantum Theory of Gravity. I. The Canonical Theory, Phys. Rev. 160, 1113-1148 (1967).

[16] Charles W. Misner, Quantum Cosmology. I, Phys. Rev. 186, 1319-1327 (1969).

[17] J. Fernando G. Barbero, Real Ashtekar Variables for Lorentzian Signature Space-times, Phys.Rev.D 51 (1995) 5507-5510, [arXiv, gr-qc/9410014.

[18] G Immirzi, The reality conditions for the new canonical variables of general relativity, Class.Quant.Grav. 10 (1993) 2347.

[19] Martin Bojowald, Loop Quantum Cosmology III: Wheeler-DeWitt Operators, Class.Quant.Grav. 18 (2001) 1055-1070; [arXiv, gr-qc/0008052.

[20] Martin Bojowald, Isotropic Loop Quantum Cosmology, Class.Quant.Grav. 19 (2002) 2717-2742; [arXiv, gr-qc/0202077].

[21] Martin Bojowald, Loop Quantum Cosmology II: Volume Operators, Class.Quant.Grav. 17 (2000) 1509-1526; [arXiv, gr-qc/9910104.

[22] Martin Bojowald, Kevin Vandersloot; Loop Quantum Cosmology, Boundary Proposals, and Inflation; [arXiv, gr-qc/0303072.

[23] Martin Bojowald, Franz Hinterleitner, Isotropic Loop Quantum Cosmology with Matter, Phys.Rev. D 66 (2002) 104003; [arXiv, gr-qc/0207038.

[24] Martin Bojowald, Loop Quantum Cosmology IV: Discrete Time Evolution, Class.Quant.Grav. 18 (2001) 1071-1088; [arXiv, gr-qc/0008053.

[25] G. Date; A Discrete Time Presentation of Quantum Dynamics, Class.Quant.Grav. 20 (2003) 303-316; [arXiv, gr-qc/0210056.

[26] Martin Bojowald, The Inverse Scale Factor in Isotropic Quantum Geometry, Phys.Rev. D 64 (2001) 084018; [arXiv, gr-qc/0105067.

[27] Martin Bojowald, Dynamical Initial Conditions in Quantum Cosmology, Phys.Rev.Lett. 87 (2001) 121301; [arXiv, gr-qc/0104072. 